\title{
Childhood hypertension: Practical approaches towards diagnosis and management
}

\author{
Vindya Gunasekera ${ }^{1}$ \\ Sri Lanka Journal of Child Health, 2012; 41(1): 29-37
}

(Key words: Childhood hypertension; diagnosis; management)

Cardiovascular disease is now the leading cause of premature mortality among adults and hypertension confers the highest attributable risk to these deaths. Since blood pressure (BP) tends to track along the same percentiles throughout life, it is accepted that children with elevated BP are more likely to become hypertensive adults ${ }^{1}$. Early identification of childhood hypertension and early intervention are crucial to reduce the cardiovascular morbidity and mortality during adulthood. Even though the importance of BP measurement in children was recognized and incorporated into the routine paediatric examination more than 2 decades ago, there are still many doubts among physcians regarding assessment and evaluation of hypertension in children. There has been significant new knowledge gained about many aspects of childhood hypertension over the past decade. This article aims to discuss the fundamentals of BP measurement and some practical approaches towards the diagnosis and management of hypertension in children based on evidence from the most updated literature.

\section{Definitions}

The definitions of hypertension in children and adolescents based on the normative distribution of BP in healthy children, (Table 1) are in accordance with the $4^{\text {th }}$ report on the diagnosis, evaluation, and treatment of high blood pressure in children and adolescents by the National High Blood Pressure Education Program Working Group ${ }^{2}$.

Table 1: Definitions ${ }^{2}$

\begin{tabular}{|c|c|}
\hline Diagnosis /Stage & Average SBP/DBP percentile for gender, age and height \\
\hline Normal & $<90^{\text {th \# }}$ \\
\hline Prehypertension & $\begin{array}{l}\text { O9 } 0^{\text {th }} \text { but }<95^{\text {th }} \text { or } \\
\left.\text { BP Ó } 20 / 80 \mathrm{mmHg} \text { (even if this is }<90^{\text {th }}\right)^{*}\end{array}$ \\
\hline Stage 1 hypertension & O9 $5^{\text {th }}$ but $<99^{\text {th }}+5 \mathrm{~mm} \mathrm{Hg}$ \\
\hline Stage 2 hypertension & O99th + $5 \mathrm{~mm} \mathrm{Hg}$ \\
\hline White-coat hypertension & $\begin{array}{l}\text { The patient has BP levels that are O5th percentile when measured in a hospital } \\
\text { or clinic but are <90th percentile outside of a clinical setting. }\end{array}$ \\
\hline
\end{tabular}

\#If systolic and diastolic categories are different, categorize by the higher value.

${ }^{*}$ This typically happens at 12 years old for SBP and at 16 years old for DBP.

$\mathrm{BP}$ records $<90^{\text {th }}$ percentile for gender, age and height are considered as normal. If the $\mathrm{BP}$ is $\mathrm{O}^{\mathrm{th}}$ percentile for gender, age and height it should be repeated twice and the average taken. Children in the pre hypertensive stage have a heightened risk for developing hypertension and those children with stage 2 hypertension have a higher risk of acute and chronic organ damage.

$\overline{{ }^{1} \text { Consultant Paediatric Nephrologist, }}$ Lady
Ridgeway Hospital for Children, Colombo

Blood pressure measurement as a routine clinical procedure ${ }^{2}$

- Children Ó̉ years old who are seen in medical care settings should have their BP measured at least once during every health care episode.

- Children <3years old should have their BP measured in following special circumstances: 
1. History of prematurity, very low birth weight or other neonatal complication requiring intensive care

2. Congenital heart disease (repaired or nonrepaired)

3. Recurrent urinary tract infections (UTI), haematuria or proteinuria

4. Known renal disease or urologic malformations

5. Family history of congenital renal disease

6. Solid-organ transplant

7. Malignancy or bone marrow transplant

8. Treatment with drugs known to raise BP

9. Other systemic illnesses associated with hypertension e.g. neurofibromatosis (NF), tuberous sclerosis (TS) etc.

10. Evidence of elevated intracranial pressure (ICP)

\section{Method for BP measurement in children}

In BP measurement, mere obtaining of a value is fruitless unless an accurate method is followed using proper devices. It is of paramount importance to use a cuff appropriate to the size of the childô upper right arm and the dimensions of the inflatable bladder underneath the cuff are very crucial. By convention, an appropriate cuff size is a cuff with a bladder width that is at least $40 \%$ of the arm circumference at a point midway between the olecranon and the acromion ${ }^{3,4,5}$. For such a cuff to be optimal for an arm, the cuff bladder length should cover $80 \%$ to $100 \%$ of the circumference of the $\operatorname{arm}^{5}$. Such a requirement demands that the bladder width-to-length ratio be at least 1:2. All the commercially available cuffs do not meet these standards. Therefore, the working group has recommended to adopt the dimensions given in table 2 when selecting an appropriate cuff. If a cuff is too small the next largest one needs to be used ${ }^{2}$.

Table 2: Recommended dimensions for BP cuff bladders

\begin{tabular}{lccc}
\hline Age range & Width $(\mathbf{c m})$ & Length $(\mathbf{c m})$ & $\begin{array}{c}\text { Maximum arm } \\
\text { circumference }(\mathbf{c m}) *\end{array}$ \\
\hline Newborn & 4 & 8 & 10 \\
Infant & 6 & 12 & 15 \\
Child & 9 & 18 & 22 \\
Small adult & 10 & 24 & 26 \\
Adult & 13 & 30 & 34 \\
Large adult & 16 & 38 & 44 \\
Thigh & 20 & 42 & 52 \\
\hline
\end{tabular}

*Calculated so that the largest arm would still allow the bladder to encircle arm by at least $80 \%$

Mercury sphygmomanometer is the standard device for BP measurement. Aneroid manometers are also quite accurate when calibrated on a biannual basis ${ }^{2}$. The popular oscillometric devices are convenient but may not be accurate, necessitating regular validation as per standard protocols ${ }^{2}$. They also tend to give higher values than auscultatory methods. Since the standard BP tables are based on $\mathrm{BP}$ values taken by the latter method, it is advised to confirm higher oscillometric values by auscultation $^{2}$. Correct technique of measuring BP using auscultation is given in figures 1 and 2 .

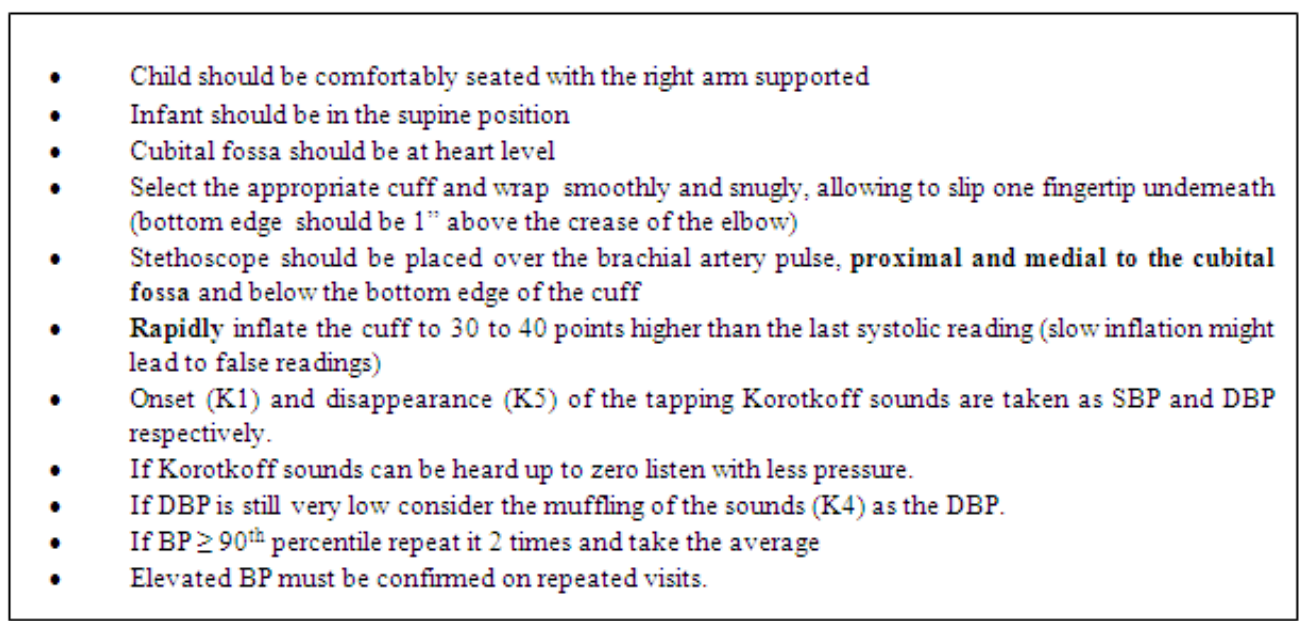

Figure 1: Key points in measuring $B P$ 

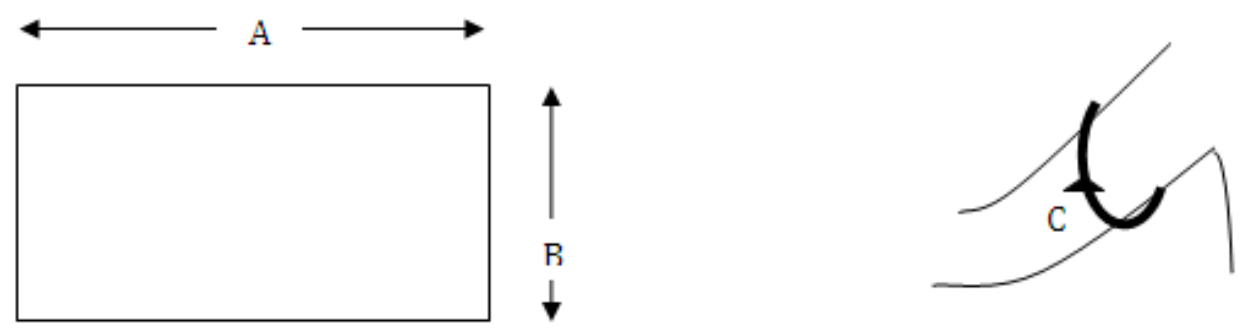

\begin{abstract}
A-Length; $\quad$ B - Width; $\quad \mathrm{C}$ - Mid am circumference
A should be approximately $80-100 \%$ of C; B should be approximately $40 \%$ of C
\end{abstract}

Figure 2. Dimensions of cuff bladder

\section{BP tables}

The most recently updated BP tables of children and adolescents are based on gender, age and height and they include the $50^{\text {th }}, 90^{\text {th }}, 95^{\text {th }}$, and $99^{\text {th }}$ BP percentiles for several differnt height percentiles. This approach avoids misclassifying children who are very tall or very short. Normative $\mathrm{BP}$ values for infants under 1 year of age are according to the Second Task Force report ${ }^{6}$ (see Figures 3-4). Defining normative blood pressure data in newborn infants is a complex task. Extremely useful data in this regard has been published by Zubrow et $\mathrm{al}^{7}$ (see figure 5A, B \& C). I have not included the normative BP tables for children $>1$ year since I assume our readers are well familiar with them.
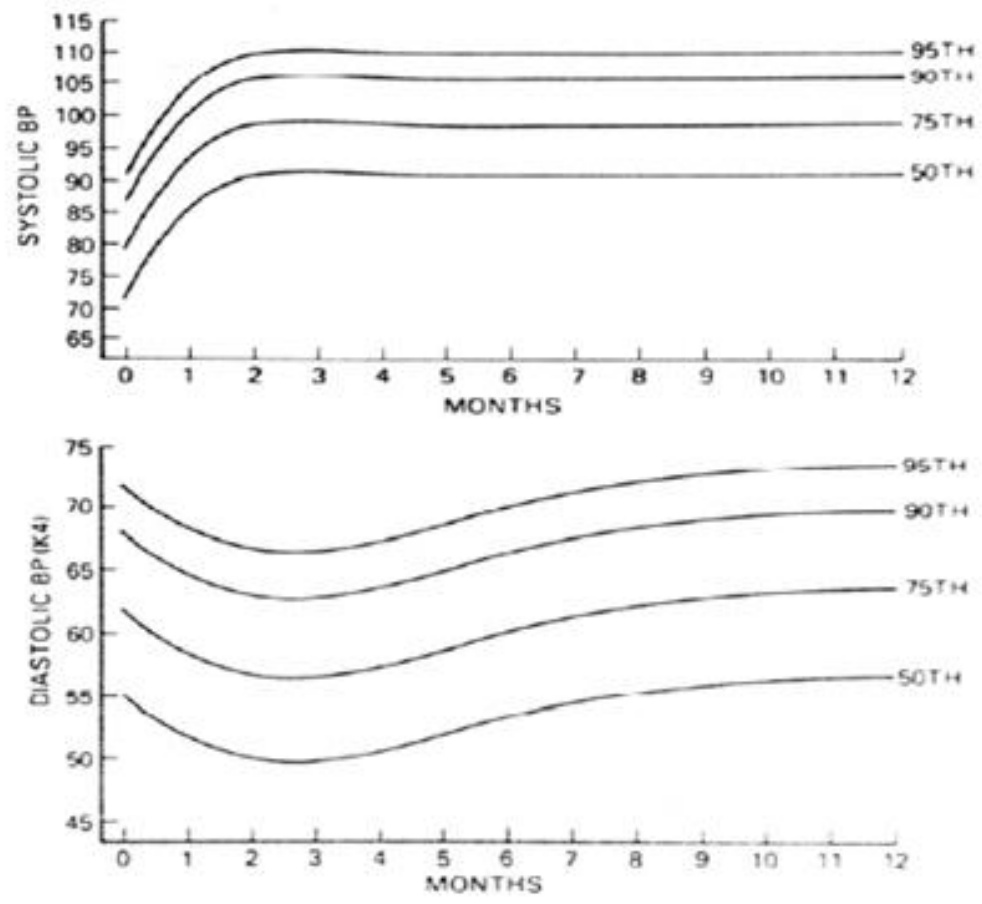

PETHCENTILE

SYSTOLIC BP

DIASTOLIC BP

HEIGHT CM

$\begin{array}{rrrrrrrrrrrrr}87 & 101 & 106 & 106 & 106 & 105 & 105 & 105 & 105 & 105 & 105 & 105 & 105 \\ 68 & 65 & 63 & 63 & 63 & 65 & 66 & 67 & 68 & 68 & 69 & 69 & 69 \\ 51 & 59 & 63 & 66 & 68 & 70 & 72 & 73 & 74 & 76 & 77 & 78 & 80 \\ 4 & 4 & 5 & 5 & 6 & 7 & 8 & 9 & 9 & 10 & 10 & 11 & 11\end{array}$

Figure 3: Boys 0-12 months age specific percentiles of clinic BP 

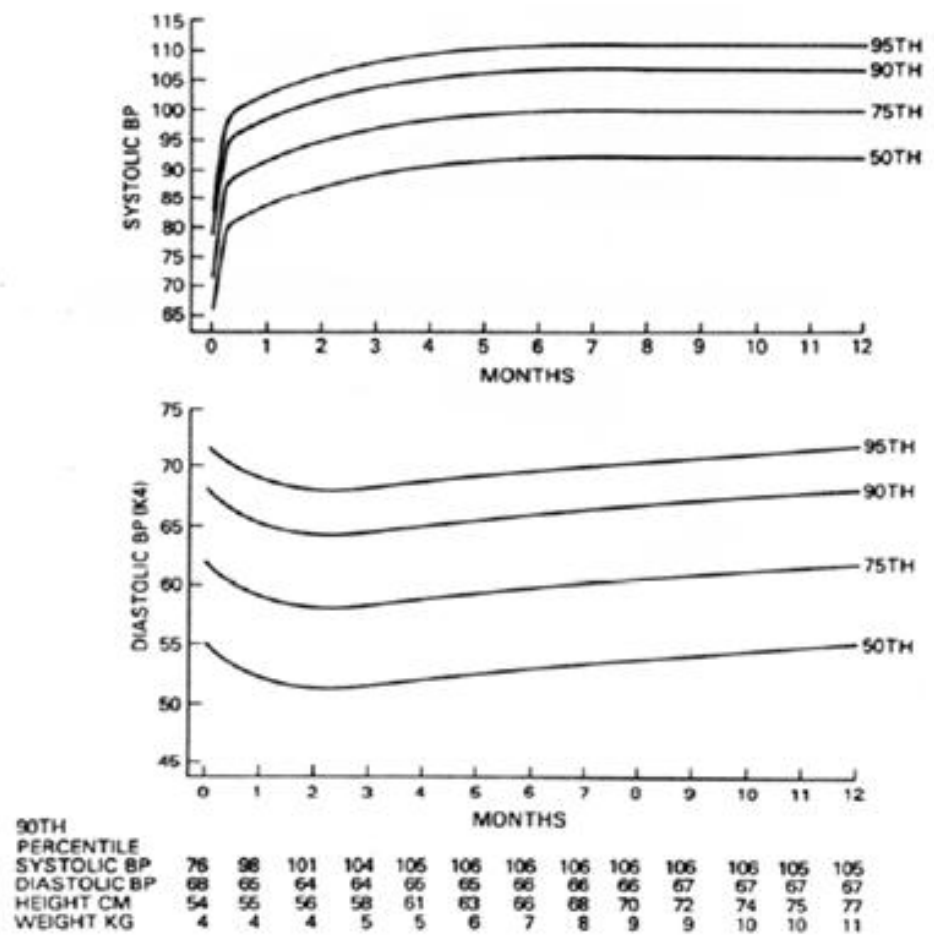

Figure 4: Girls 0-12 months age specific percentiles of clinic BP
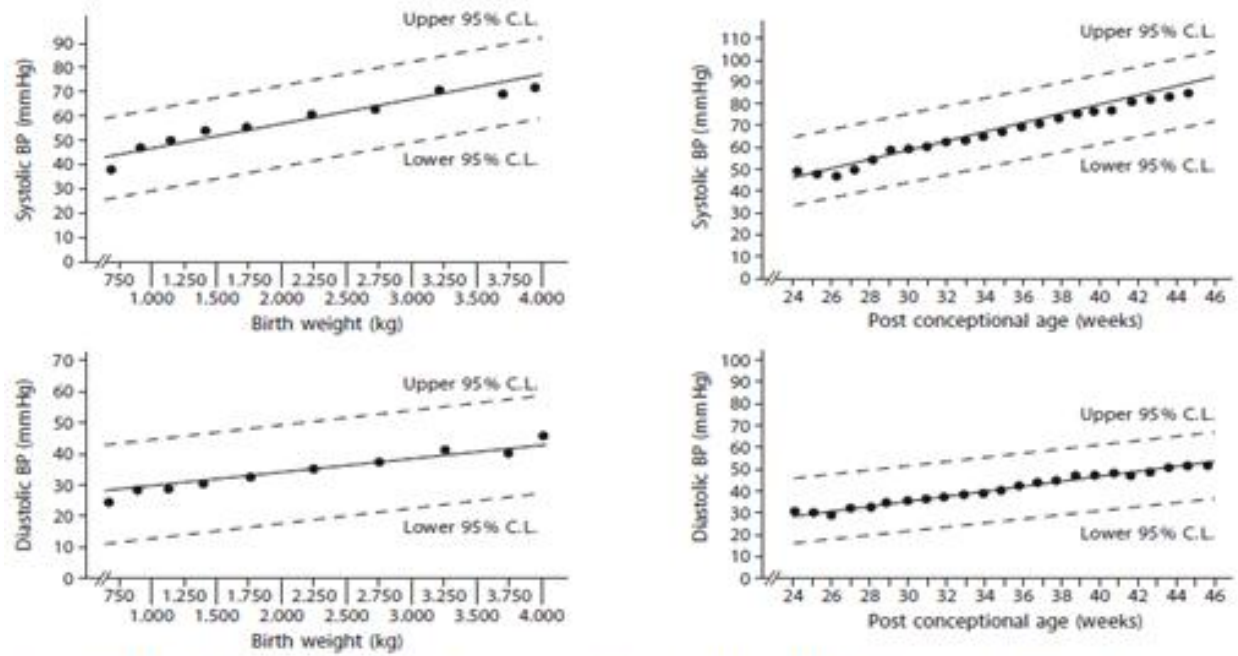

Figure 5A: Linear regression of mean systolic and diastolic blood pressures by birth weight on day 1 oflife, with $95 \%$ confidence limits (upper and lower dashed lines).

Figure 5B: Linear regression of mean systolic and diastolic blood pressures by postconceptual age in weeks, with $95 \%$ confidence limits (upper and lower dashed lines). 

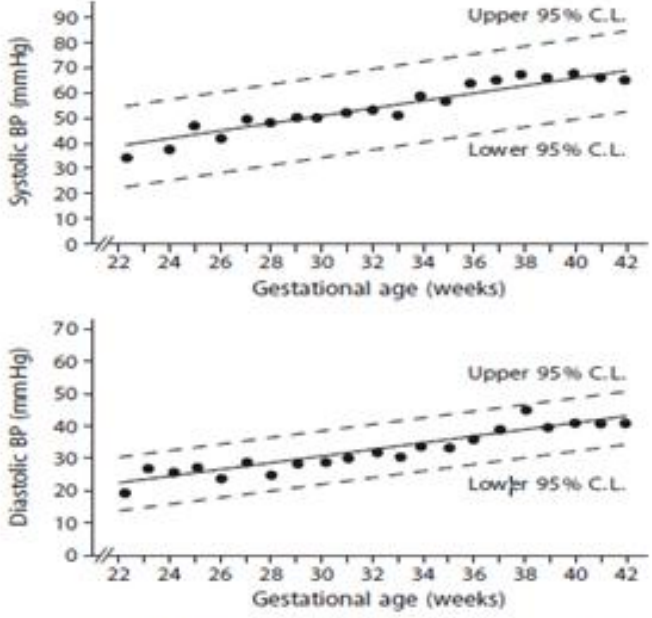

Figure 5C: Linear regression of mean systolic and diastolic blood pressures by gestational age on day 1 of life, with $95 \%$ confidence limits (upper and lower dashedlines).

\section{What is ambulatory blood pressure monitoring ${ }^{8}$ (ABPM)?}

$\mathrm{ABPM}$ is a non-invasive method of monitoring $\mathrm{BP}$ over a fixed time interval, usually 24 hours. It involves a BP cuff attached to a small portable monitor. BP recordings are automatically obtained at prefixed time intervals and recorded by the monitor. Hypertension can be determined based on several indices calculated using these data. ABPM is especially helpful in the evaluation of whitecoat hypertension, risk for hypertensive organ injury, apparent drug resistance, hypotensive symptoms with antihypertensive drugs and BP patterns in special conditions such as episodic hypertension, chronic kidney disease (CKD), diabetes mellitus (DM) and autonomic dysfunction.

\section{Common causes of of secondary hypertension in children}

\section{Classification by aetiology $y^{9,10}$}

1. Renal parenchymal disease (RPD)

Congenital anomalies: polycystic kidney diseases, hypoplastic or dysplastic kidnyes and other structural anomalies

Glomerulonephritis

Haemolytic uraemic syndrome

Renal parenchymal scarring associated with recurrent UTI, reflux disease, renal injury, trauma and renal vein thrombosis (RVT)

Chronic renal failure (CRF)

Intrarenal tumours (Wilms tumour, renal cell carcinoma)

2. Renovascular disease (RVD)

Fibromuscular dysplasia (majority), Vasculitis, Thrombosis,

Mid-aortic syndrome or abdominal coarctation, Associated with NF, TS, Williams, Turner,

Marfans,

Klippel-Trenaunay-Weber and Epidermal naevus syndrome

3. Coatctation of aorta (COA)

4. Endocrine diseases

Mineralocorticoid excess

Corticosteroid excess

Hyperthyroidism

Catecholamine excess

Hyperparathyroidism

5. Genetic (Monogenic)

eg: Liddleôs syndrome, Gordonôs syndrome, Glucocorticoid remediable aldostronism (GRA), apperant minerelocorticoid excess etc

6. Neurological

Increased ICP

Familal dysautonomia

Guillain-Barre syndrome

7. Drugs

8. Obstructive sleep apnoea (OSA)

RVD causes some $10 \%$ of all childhood (below 18 years of age) hypertension. It is important to diagnose early since it is potentially amenable to angioplasty or surgery.

\section{Common causes of secondary hypertension by age $^{9,10,11}$}

(see table 3)

Table 3: Common causes of secondary hypertension by age

\begin{tabular}{|l|l|l|l|l|}
\hline \multicolumn{1}{|c|}{ New born } & \multicolumn{1}{|c|}{ First year } & \multicolumn{1}{c|}{ 1-6 years } & \multicolumn{1}{c|}{ 6-12 years } & \multicolumn{1}{c|}{ 18 years } \\
\hline RVD e.g. renal & COA & RPD & RPD & Essential \\
artery thrombosis, & RVD & RVD & RVD & hypertension \\
renal artery & RPD & COA & Essential & hypertension \\
stenosis, renal vein & Iatrogenic causes & & COA & RVD \\
thrombosis & Tumours & & & \\
Congenital RPD & & & & \\
Acquired RPD & & & & \\
COA & & & \\
\hline
\end{tabular}

Note: This table covers only the common causes for each age group. 


\section{Hypertension of the newborn}

RVD is the commonest cause of hypetension in the newborn period. Several predisposing factors for renovascuar accidents in the newborn are identified and a clear association between use of umbilical arterial catheters and development of arterial thrombi has been demonstrated ${ }^{11}$. The next largest group comprises of RPD mainly cystic kidney diseases and other structural anomalies of the kidneys ${ }^{11}$. Invasive laboratory investigstions can be largely avoided in the majority of these neonates with a careful history and examination.

\section{Basic diagnostic investigations}

Basic investigations can be catergorized into 3 groups $^{10}$

1. Evaluation for cause

Blood urea (BU), serum creatinine ( $\mathrm{SCr}$ ), serum electrolytes (SE), full blood count (FBC), urinalysis (UFR), renal ultrasound (USS) with doppler, chest $x$-ray (CXR), electrocardiogram (ECG), 2 dimensional echocardiogram (2D Echo)

2. Evaluation for co-morbidity

Fasting lipid, fasting blood sugar, serum uric acid, fasting insulin (obese children)

Glucose tolerance test (GTT), glycated haemoglobin (HbAlc) if there is strong family history of diabetes mellitus (DM)

Polysomnography if history is suggestive of obstructive sleep apnoea (OSA)

3. Evaluation for evidence of target organ damage (TOD)

Urine micro-albumin, ECG, 2D Echo

\section{Children who need to be evaluated more completely}

1. Very young child

2. With stage 2 hypertension

3. History and examination favouring a secondary cause

4. If ABPM shows a secondary pattern

5. Evidence of TOD

\section{Practical approaches for diagnosis ${ }^{2,9,10}$}

\section{Clues for diagnosis of common aetiologies}

\section{RPD}

a. Most urological or glomerular causes can be strongly suspected by history and examination e.g. history of UTI, voiding dysfunction, glomerular nephritis (GN), haematuria, proteinuria

b. This can be confirmed by basic investigations $B U, S E, S C r, U F R,{ }^{*}$ urine protein,

USS and abdominal $x$-ray $(K U B)$ : to rule out congenital anomaly or disparate renal size

("Proteinuria can be secondary to RPD or hypertension itself)

c. Further imaging, biochemical and histological investigations have to be decided on an individualized basis

\section{RVD}

Symptoms and signs suggestive of $R V D$

a. Very high blood pressure.

b. Secondary symptoms of high blood pressure including cerebral symptoms, cardiac failure and facial palsy

c. Difficult to treat hypertension that is not well controlled on full doses of at least two antihypertensive drugs.

d. Diagnosis of a syndrome with a higher risk of vascular disease

e. Signs of vasculitis in particular Takayasu disease.

f. Known or suspected previous vascular insult such as renal artery thrombosis or umbilical artery catherisation.

g. Transplanted kidneys

h. Bruit heard over the artery/ies.

i. *Polycythaemia

j. Elevated peripheral plasma renin or moderate hypokalaemia.

*Also seen with OSA

It is important to remember that a large proportion has intra-renal small vessel disease in addition to main artery disease and also stenosis of other vessles including aorta, visceral arteries and cerebral vessles.

\section{Specific investigations for RVD}

a. Plasma renin activity high in 70-80\% of renal artery stenosis (RAS) - both supine and erect values need to be taken for better comparison

b. Doppler USS - can miss disease in segmental intra-renal arteries

c. Captopril DTPA/DMSA and/or MAG 3 scan 60-90\% sensitivity and specificity but unreliable with bilateral disease and can miss disease in intra-renal arteries

d. MR angiogram or CT angiogram Good for main arteries but may miss small intra-renal vessels especially in infants

e. Digital substraction angiography or classical arteriography - the gold standard

\section{Aldosterone excess}

Features of primary hyperaldosteronism (PHA)

a. **Low potassium

b. Metabolic alkalosis 
c. Low 24 hour urinary sodium

d. High 24 hour urinary potassium

e. Usually modertate to severe hypertension refractory to therapy

f. Low renin \& high aldosterone **potassium can also be low in secondary hyperaldosteronism and monogenic hypertension (but not in Gordon syndrome )

\section{Specific hormone tests}

a. Renin profiling (supine and erect) High in RVD and RPD (also dehydration) Low in PHA, excess glucocorticoid \& monogenic hypertension (including glucocorticoid remediable aldosteronism or GRA)

b. Aldosterone High in RVD (85\%), PHA and GRA

Low in excess glucocorticoid and monogenic hypertension except GRA

c. Confirmatory tests of PHA - Saline suppression test, oral salt loading test

d. Plasma \& urine VMA, HVA, adrenaline, noradrenaline

e. Plasma and urine steroid levels

Important facts in hormone testing

a. Hormones are extremely labile

b. Blood levels massively fluctuate

c. Tests are very costly and patients should be carefully selected for each investigation

d. Patients need to be prepared as per specific instructions

e. Specific precautions should be adhered to while collecting \& transporting

\section{Principles of investigating renin angiotensin} aldosterone system

a. Correct hypokalaemia and dehydration before testing

b. Avoid drugs that alter aldosterone or renin secretion (if possible)

c. Withdraw beta-blockers and spironolactone for 2-4 weeks.

Beta-blockers lower renin secretion and spironolactone inhibits mineralocorticoid receptor, thereby increasing renin secretion. If the screening test is performed while on ACE inhibitors, angiotensin receptor blockers, calcium channel antagonists, or alpha-blockers, and aldosterone levels remain frankly elevated in the setting of suppressed renin activity, the likelihood of PHA remains high

\section{Other special investigations}

a. MIBG or In-octreotide scan for phaeochromocytoma

b. CT or MRI for adrenal or other tumours

c. Genetic studies for monogenic hypertension

\section{Essential hypertension}

Essential hypertension is an emerging major problem in adolescents and it is the commonest cause of hypertension in this age group. It is considered as part of the metabolic syndrome associated with obesity, juvenile diabetes, hyperlipidaemia and family history of hypertension.

\section{Treatment of confirmed hypertension ${ }^{2,10,12,13}$}

\section{Pre hypertension}

1. Life style modifications with non pharmacologocal methods

- Weight management

- Physical activity

- Diet counselling

2. Re-evaluate in 4-6 months

3. Those with co-morbidities or TOD needs more intense approach \pm drug therapy

\section{Stage 1 Hypertension}

1. Life style modifications

2. Treat if:

- Symptomatic

- With CKD, DM

- Secondary Hypertension

- High risk of cardiovascular disease (CVD)

- Family history of premature CVD (men $<55$, women $<65$ )

- $T O D$

- $\quad$ persistent hypertension despite life style modification

\section{Stage 2 hypertension}

1. Admit

2. Treat \& investigate promptly

\section{Antihypertensive drug therapy Principles}

1. The choice of the initial drug depends on the patient as well as physician's choice

2. Specific classes of antihypertensive drugs should be used in specific clinical circumstances

- $\quad A C E I \& A R B s$ in DM with microalbuminuria or proteinuric renal diseases

- Beta-blockers or calcium channel blockers in migraine

- Diuretics for fluid overloaded conditions 
3. Start at the lowest recommended dose.

4. Increase to the maximum dose before adding another unless the patients gets side effects

5. Monitor for side effects e.g. monitor serum electrolytes and creatinine while on diuretics and ACEI

\section{BP Goal}

1. Uncomplicated primary hypertension with no TOD - maintain $<95^{\text {th }}$ percentile $^{2}$

2. CKD, DM, TOD- maintain $<90^{\text {th }}$ percentile $^{2}$

3. Intensified BP control $<50^{\text {th }}$ percentile in glomerulopathies and renal dysplasias have improved renal outcome ${ }^{13}$

\section{Management of hypertensive urgencies and emergencies}

\section{Hypertensive urgencies}

Definition: Significant elevation of BP with symptoms of nausea, headache and blurred vision and without acute end-organ injury. (More common in the paediatric population)

\section{Hypertensive emergencies}

Definition: Elevation of both SBP and DBP with acute end-organ injury like cerebral infarction, cerebral haemorrhage, encephalopathy, left heart failure, grades III-IV retinopathy (exudates, haemorrhage, papilloedema. (Patients are symptomatic with complaints of headaches, epistaxis, blurred vision, nausea and vomiting)

\section{General principles}

1. Monitor vital signs

2. Order basic investigations

3. Arrange a CT-scan of the brain if neurological signs are positive

4. Consider sedation and pain releif

5. Start oral short acting nifedipine while preparing parenteral drugs (Note:Sublingual nifedipne can cause a drastic BP drop)

\section{Drug therapy for hypertensive emergencies}

1. Need parenteral drugs

2. Decrease BP by $1 / 3$ over the first $6-12$ hours after presentation, and then gradually normalize the BP over next 24 to 48 hours

3. The goal is to reduce BP to a safe level to limit further end-organ damage.

4. Initial rapid decrease may be harmful due to disrupted cerebral auto-regulation

5. Frequent blood pressure monitoring
6. Neuro-observations and pupillary reactions

7. Aim to reduce blood pressure slowly

8. Patient needs at least 2 large bore iv cannulae

9. Ensure that an intravenous fluid bolus can be given if BP drops acutely

Drug therapy for hypertensive urgency

1. The goal is to reduce BP within a 24-hour period.

2. Oral or parenteral therapy can be given

Anti-hypertensive medications in hypertensive urgencies or emergencies

1. Oral short acting nifedipine

2. IV labetalol - has favourable CVS and CNS effects (can use with raised ICP)

3. IV GTN - reduce coronary spasm

4. IV hydralazine

5. IV nitroprusside

6. IV diazoxide

7. IV nicardipine - less CVS side effects than nifedipine

8. IV phentolamine - drug of choice for catecholamine excess

9. IV frusemide and IV bumetanide $\ddot{I}$ drugs of choice in glomerular disease associated with fluid overload

\section{Summary}

Clinicians caring for children \& adolescents should familiarize themselves with current practices of managemnt of hypertension in this age group and incorporate them into their clinical decisionmaking. Early and accurate diagnosis, appropriate referral and timely intervention and follow up of these patients will reduce acute morbidity as well as future burden of adult cardiovascular morbidites and mortalities related to hypertension.

\section{References}

1. Erlingsdottir A, Indridason OS, Thorvaldson $\mathrm{O}$, Edvardsson VO. Blood pressure in children and target-organ damage later in life. Pediatr Nephrol 2009.

http://dx.doi.org/10.1007/s00467-009-1350

2. National High Blood Pressure Education Program Working Group on High Blood Pressure in Children and Adolescents. The fourth report on the diagnosis, evaluation, and treatment of high blood pressure in children and adolescents. National Heart, Lung, and 
Blood Institute, Bethesda, Maryland. Pediatrics 2004; 114:555ї 76.

http://dx.doi.org/10.1542/peds.114.2.S2.555

3. Gomez-Marin O, Prineas RJ, Rastam L. Cuff bladder width and blood pressure measurement in children and adolescents. J Hypertens 1992; 10:1235i 41.

http://dx.doi.org/10.1097/00004872199210000 $\underline{-00018}$

4. American Heart Association. Home monitoring of high blood pressure. Available at: www.americanheart.org/presenter.jhtml?identi fier=576. Accessed March 18, 2004

5. National High Blood Pressure Education Program Working Group on hypertension control in children and adolescents. Update on the 1987 Task Force Report on high blood pressure in children and adolescents: a working group report from the National High Blood Pressure Education Program. Pediatrics 1996; 98:649ї 58.

6. Task Force on Blood Pressure Control in Children. Report of the Second Task Force on Blood Pressure Control in Childrend 1987. National Heart, Lung, and Blood Institute, Bethesda, Maryland. Pediatrics 1987; 79:1ї 25.

7. Zubrow AB, Hulman S, Kushner H, Falkner B. Determinants of blood pressure in infants admitted to neonatal intensive care units: a prospective multicenter study. J Perinatol 1995; 15: 470 ï 9.
8. Oổrien E, Coats A, Owens P, et al. Use and interpretation of ambulatory blood pressure monitoring: recommendations of the British hypertension society. BMJ 2000; 320:1128ї 34. http://dx.doi.org/10.1136/bmj.320.7242.1128

9. Nehal US, Ingelfinger JR. Paediatric hypertension: recent literature. Current Opinion in Pediatrics 2002, 14:189ї 96. http://dx.doi.org/10.1097/00008480200204000 $\underline{-00008}$

10. Leung LCK. Hypertension: Diagnosis and evaluation. In: Chiu MC, Yap HK editors. MedcomLtd; 2005. Practical paediatric nephrology. An update of current practice. $\mathrm{P}$ 80-88.

11. Flynn JT. Neonatal hypertension: diagnosis and management. Pediatr Nephrol 2000; 14:332ï 41 . http://dx.doi.org/10.1007/s004670050771

12. Chobanian AV, Bakris GL, Black HR, et al. The seventh report of the joint national committee on prevention, detection, evaluation, and treatment of high blood pressure: the JNC 7 report. JAMA 2003; 289: 2560 ï 72.

http://dx.doi.org/10.1001/jama.289.19.2560

13. The ESCAPE Trial Group. Strict bloodpressure control and progression of renal failure in children. $N$ Engl J Med 2009; 361:1639-50.

http://dx.doi.org/10.1056/NEJMoa0902066 